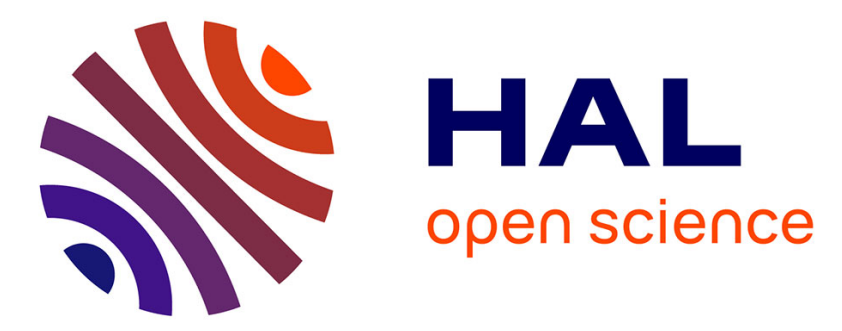

\title{
Typologie d'un geoweb des flux et réseaux
}

Françoise Bahoken, Grégoire Le Campion, Marion Maisonobe, Laurent Jégou, Etienne Come

\section{To cite this version:}

Françoise Bahoken, Grégoire Le Campion, Marion Maisonobe, Laurent Jégou, Etienne Come. Typologie d'un geoweb des flux et réseaux. Geomatica, 2020, pp.48. 10.1139/geomat-2020-0007 . hal02997827

\section{HAL Id: hal-02997827 \\ https://hal.science/hal-02997827}

Submitted on 10 Nov 2020

HAL is a multi-disciplinary open access archive for the deposit and dissemination of scientific research documents, whether they are published or not. The documents may come from teaching and research institutions in France or abroad, or from public or private research centers.
L'archive ouverte pluridisciplinaire HAL, est destinée au dépôt et à la diffusion de documents scientifiques de niveau recherche, publiés ou non, émanant des établissements d'enseignement et de recherche français ou étrangers, des laboratoires publics ou privés. 


\section{Typologie d'un geoweb des flux et réseaux}

\section{Bahoken Françoise $^{13}$, Le Campion Grégoire ${ }^{2}$, Maisonobe Marion ${ }^{3}$ Jégou Laurent $^{4}$, Côme Étienne ${ }^{5}$}

1. Univ Gustave Eiffel / IFSTTAR - AME, Cité Descartes, 14-20 Boulevard Newton, F-77420 Champs-sur-Marne, France et UMR 8504 Géographie-Cités / Équipe PARIS / CNRS, Campus Condorcet, Bâtiment de recherches sud, 5, cours des Humanités, 93322 Aubervilliers, France, francoise.bahoken@univ-eiffel.fr

2. UMR 5319 Passages, Équipe ARD / CNRS, Maison des Suds, 12 Espl. des Antilles, F-33600 Pessac, France, Gregoire.LECAMPION@cnrs.fr

3. UMR 8504 Géographie-Cités / Équipe PARIS / CNRS, Campus Condorcet, Bâtiment de recherches sud, 5, cours des Humanités, 93322 Aubervilliers, France, marion.MAISONOBE@cnrs.fr

4. UMR 5193 LISST / Université de Toulouse 2 / CNRS, Maison de la Recherche, 5, allées AntonioMachado, F-31058 Toulouse Cedex 9, France.jegou@univ-tlse2.fr

5. Univ Gustave Eiffel / IFSTTAR - COSYS, Cité Descartes, 14-20 Boulevard Newton, F-77420 Champs-sur-Marne, etienne.come@univ-eiffel.fr 


\section{Typologie d'un geoweb des flux et réseaux}

$\boldsymbol{R E S U M E}$. L'analyse de la dynamique des aires urbaines ou des métropoles, la délimitation de leurs aires fonctionnelles et la comparaison spatio-temporelle de leurs motifs est souvent freinée par l'insuffisance de données relationnelles (portant sur des liens entre des entités) ouvertes et l'absence jusque récemment de dispositifs d'analyse et de géo-visualisation dédiés. Au-delà des questions d'ouverture des données (géo)numériques, nous proposons un panorama du geoweb, le processus de création de cartes dans le contexte du web 2.0, spécifique aux flux et réseaux localisés. L'éclairage ainsi apporté sur les pratiques cartographiques actuelles révèle trois grandes familles d'applications web ainsi que les besoins d'une communauté, restreinte mais dynamique, d'analyser librement ses propres jeux de données.

MotS-CLÉS : geoweb, flux, réseaux, application web, cartographie thématique.

ABSTRACT. Analysing the dynamics of urban areas or metropolises, delineating their functional areas and comparing their spatio-temporal patterns is often limited by the lack of open relational data (on links between entities) and the absence until recently of dedicated analysis and geovisualization frameworks. Beyond the questions of opening (geo)digital data, we propose a panorama of a geoweb, the process of creating maps in the context of the Web 2.0, specific to flows and networks. The insights provided on current mapping practices reveal three main families of web applications, as well as the needs of a small but dynamic community to freely analyze its own data sets.

KEYWORDS: geoweb, flows, spatial networks, web applications, thematic cartography. 


\section{Introduction}

L'analyse de la dynamique des aires urbaines ou des métropoles, la délimitation de leurs aires fonctionnelles, la comparaison spatio-temporelle de leurs motifs est souvent freinée par deux catégories de problèmes inhérents aux données et aux outils. L'insuffisance de données ouvertes décrivant interactions et interrelations territoriales, pourtant cruciales pour l'aménagement du territoire et la planification, freine en effet les possibilités d'analyses empiriques. L'absence de dispositifs d'analyse et de géo-visualisation dédiés a d'ailleurs pu contribuer à éluder du paysage cartographique actuel nombre d'images présentant pourtant un intérêt visuel et analytique fort (Bahoken, 2016).

Cet article est la première contribution issue du projet interdisciplinaire : gflowiz (geographic flow visualisation $)^{1}$. Ce projet est structuré autour de deux objectifs principaux : d'une part, la constitution et l'analyse d'un corpus d'applications de web (carto)graphie de données décrivant des mobilités et relations spatio-temporelles; d'autre part, le développement d'Arabesque ${ }^{2}$, une application web d'analyse et de géo visualisation de flux, de réseaux et de mouvements. Au-delà des questions liées au partage des données relationnelles (portant sur des liens entre entités) qui relèvent de problèmes spécifiques, cet article propose :

- la constitution d'un corpus d'applications de web (carto)graphie, ouvert et librement accessible en ligne sous la forme d'un tableau de bord interactif ${ }^{3}$;

\footnotetext{
1 https://geoflowiz.hypotheses.org/

2 http://arabesque.ifsttar.fr/

3 https://analytics.huma-num.fr/Gregoire.LeCampion/dashboard gflowiz/\#section-base-de-donnees
} 
- l'analyse préliminaire de ce corpus selon des méthodes multicritères, conduisant à une première typologie ;

- la création, la mise en ligne et l'analyse des résultats d'une enquête menée auprès d'utilisateurs potentiels pour identifier les besoins en vue du développement d'une nouvelle application dédiée ;

- l'introduction de l'application cartographique en ligne issue de ces réflexions, Arabesque.

Notre fondons notre hypothèse principale sur l'engouement suscité depuis les années 2000 par le geoweb, une notion apparue aux alentours de 2005 (Haklay et al., 2008) et précisée par T. Joliveau et al. (2010 et 2011) comme le processus de création de cartes dans le contexte du web 2.0 (Crampton, 2009, Merickskay et Roche, 2011), fondé sur la participation (Turkucu et Roche, 2008), l'expérience utilisateur (Sui et al., 2012) et la compétence informatique. Nous considérons que cette informatisation générale de la société (cf. Goodchild, 2007) est susceptible de profiter à l'analyse cartographique des flux et des réseaux localisés. En particulier, l'ouverture des possibilités de stockage et de traitement interactif des données massives est en mesure d'étendre les possibilités d'analyse et de représentation géo(carto)graphique de ces données.

Le support web a en effet entraîné un renouvellement général de la fabrique cartographique qui a permis d'introduire de nouvelles possibilités de représentation des changements (Cunty et al., 2017). Les possibilités d'animation autorisent une visualisation moins naïve des mouvements, celles d'interactions et de cartographies dynamiques admettant une exploration active des informations facilitant leur appropriation. Ces nouvelles fabriques, qui conduisent à 
la production de nouvelles images cartographiques numériques (Joliveau et al., 2013), présentent bien sûr un risque d'uniformisation (Noucher, 2017), mais elles participent avant tout au renouvellement des usages de la carte et à redéfinir la figure du cartographe (Zanin, 2013), à moins d'en introduire une nouvelle : l'utilisateur-concepteur.

Les matrices et listes de liens décrivant interrelations et interactions sont largement mobilisées dans l'analyse des réseaux sociaux, localisés ou non, ou de transport. Elles donnent plus particulièrement lieu à des représentations sous la forme de matrices ordonnables et de graphes (Berhisch et al., 2016), moins de cartes. Mis à part le fait que les productions réalisées ne sont pas toujours pas convaincantes ou utiles (Baron et al., 2012), les risques d'uniformisation apparaissent préoccupants. Ils tiennent au fait que les utilisateurs non avertis hésitent à s'éloigner des paramètres, des choix de visualisation, voire des méthodes d'analyse proposés par défaut dans les outils qu'ils utilisent. Même si ces outils sont aujourd'hui plus nombreux et plus accessibles, ils restent caractérisés par des modalités de visualisation peu variées et difficiles à paramétrer de manière fine. Dans le domaine de la visualisation des graphes, par exemple, nous pouvons citer le cas du choix imposé de l'algorithme de partitionnement de Louvain et de son paramètre de résolution fixé par défaut dans la plupart des logiciels (Beauguitte, 2019). La tendance à opter pour le mode de représentation et d'analyse le plus accessible («à portée de clic ») peut, dans certains cas, s’expliquer par une méconnaissance des enjeux théoriques et méthodologiques associés aux choix de représentation du concepteur, pour ce qui relève de son intention - choix du mode de représentation, éventuel choix d'une méthode de discrétisation ou de traitement des données, choix des signes et des figurés, choix des couleurs et autres variables rétiniennes- et des effets de ces choix sur la réception des images par différents publics (Bahoken et al., 2020). 
Compte tenu de ces risques et des nouvelles possibilités technologiques, nombreux sont les spécialistes à reconnaître actuellement qu'une meilleure connexion entre développeurs d'applications du geoweb, thématiciens, géomaticiens, cartographes positionnés à l'interface de ces deux communautés, pourrait se révéler fructueuse. Le développement d'une algorithmique dédiée, en réponse à des questionnements spécifiques, combinant informations statistiques, géographiques et cartographiques, incluant des aspects de sémiologie pour améliorer leur lisibilité, pourrait conduire à renouveler l'offre de production visuelle contemporaine.

Dans un premier temps nous présentons un panorama du geoweb des flux et réseaux originedestination (Section 2). Il révèle, dans un second temps, trois grandes familles d'applications (Section 3), ouvrant la voie à une enquête à propos des desiderata pour une nouvelle application (Section 4). La typologie des applications présentée dans ce premier article de notre collectif traite essentiellement des caractéristiques d'ordre thématiques, méthodologiques et cartographiques des applications inventoriées; les variables technologiques de ces applications feront l'objet d'une prochaine contribution.

\section{Un corpus unique d'applications de web cartographie}

Dans le cadre du projet gflowiz, nous avons commencé par réaliser un état de l'existant, c'est-à-dire par recenser un ensemble assez varié et aussi large que possible d'applications relevant du geoweb des flux et réseaux localisés dans un espace géographique. La construction de la liste d'applications est décrite au paragraphe suivant. Le corpus ainsi constitué recense près de 80 applications web décrites par 46 variables. Ces variables correspondent aux différentes caractéristiques des applications que nous avons déterminées comme intéressantes 
par rapport à nos objectifs : étudier les besoins et produire une application ouverte et adaptée. Ainsi, nous nous sommes attelés à établir un état de l'art, recenser les différents sujets et publics d'utilisation de ces outils, les types de représentation présentés, les techniques et les algorithmes mobilisés, leur degré de réemploi possible ainsi que leur qualité logicielle. Ce premier travail de recension a permis d'observer que la production cartographique en ligne - visant à représenter a connu une hausse considérable au cours des dernières années (voir Fig. 2). Sachant que l'une des raisons régulièrement évoquées pour justifier le caractère secondaire de la carte de flux, comme modalité de représentation des données relationnelles, est généralement d'ordre technologique (en raison du coût d'accès aux outils existants pour les non-initiés) sans compter les problèmes de méthodes, l'hypothèse que le développement d'une application geoweb spécifique, visant à rendre plus accessible la production de carte de flux en ligne, puisse faciliter les choses est donc apparue plus que séduisante à l'issue de cet inventaire.

\subsection{Constitution du corpus et possibilités d'exploration}

Un inventaire large de sites web a été réalisé en réponse à la question suivante : Quelles sont sur le web les possibilités actuelles de géo-visualisation de flux, trafics et mouvements?

Deux phases de collecte sont remarquables. La première d'entre elles, réalisée en 2009 relève de la veille générale via les moteurs de recherche de Google et Google Scholar. Les applications identifiées lors de cette première phase de collecte ont fait l'objet d'une recension en ligne ${ }^{4}$, mais la curation effectuée ne saurait être considérée comme régulière. Il faudra en effet attendre 2015 pour cela, avec une reprise de la veille sur Twitter, ScoopIt, Google, Google Scholar, sur la base de mots-clés spécifiques (flowmap, trade flows, migrations flows) avec une

\footnotetext{
4 https://www.scoop.it/t/analyse-des-matrices-de-flux
} 
sauvegarde systématique du lien (et des images) vers les applications identifiées sur le réseau social SeenThis ${ }^{5}$. Les 80 applications ainsi collectées de 2017 à janvier 2019 ont alors fait l'objet d'un examen minutieux à l'aide d'une première grille de lecture permettant leur classement manuel en six grands thèmes ainsi définis : A- Contexte et sujet ; B- Type de représentation principale; C- Qualité logicielle; D- Public visé; E- Technologies mobilisées et FMétadonnées. L'exploration de la liste d'applications obtenue a ensuite été rendue possible grâce au développement d'une première version d'un tableau de bord interactif réalisé sous R/Shiny, initialement réalisé dans le cadre du stage de fin d'études de A. Nguyen (2018), enrichie et désormais maintenue (Le Campion et Nguyen, 2019).

La première grille de lecture comprenant 46 variables est apparue trop détaillée pour permettre son exploitation simple. Des regroupements ont par la suite été réalisés, donnant lieu à une seconde version du tableau de bord actuellement en ligne ${ }^{6}$. La fabrique cartographique spécifique au geoweb peut ainsi être explorée à l'aide d'un double système de filtrage interactif (voir Fig. 1).

\footnotetext{
5 https://seenthis.net/people/fbahoken

6 https://geoflowiz.hypotheses.org/dashboard-des-applications
} 


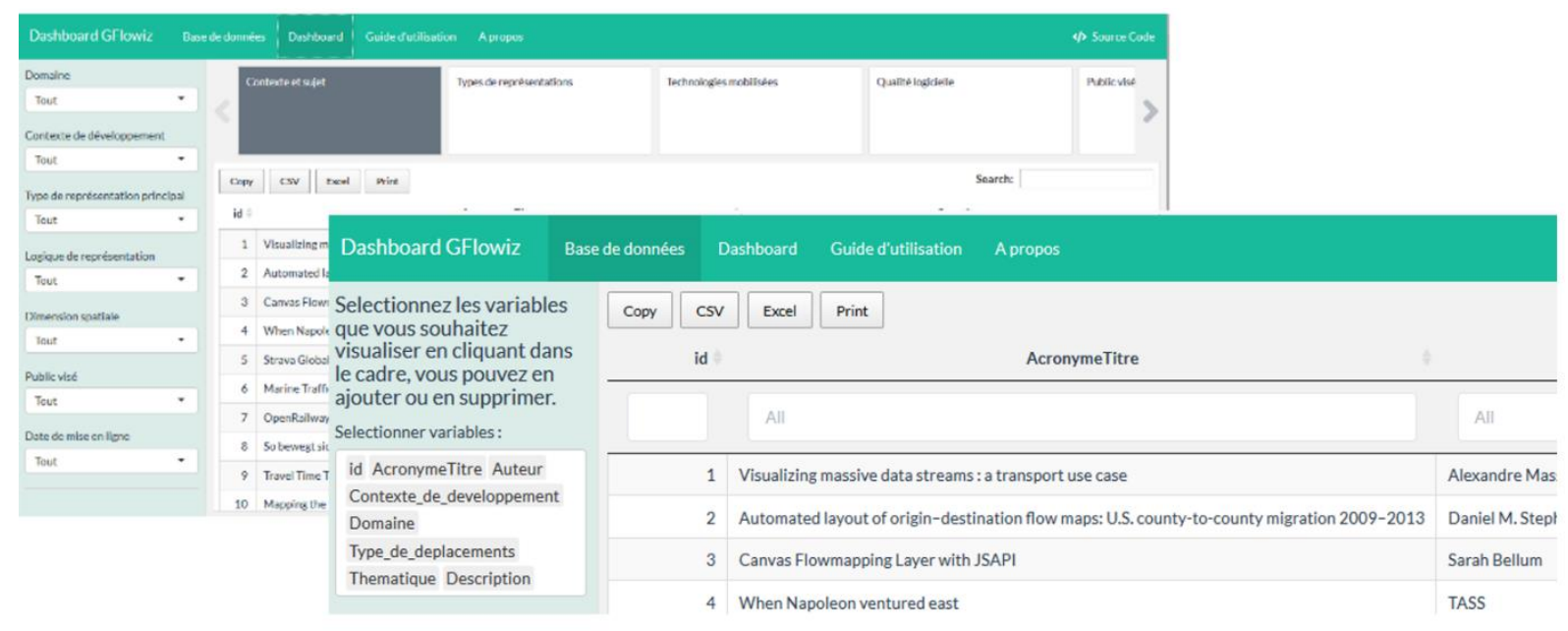

Source : Projet geographic flow vizualisation, gllowiz (2019), Tableau de bord des applications recensées

(https://geoflowiz hypotheses.org/dashboard-des-applications)

FIGURE 1. Tableau de bord des applications recensées

Une première possibilité de filtrage est accessible sur la partie latérale gauche du tableau interactif, elle permet de sélectionner la ou les variables à visualiser. La seconde possibilité de filtrage proposée permet une exploration résultant du croisement entre une sélection de variables (domaine, contexte de développement, type de représentation principale, etc.) et quelques grands thèmes de la grille.

\subsection{Description du corpus d'applications}

La fabrique cartographique spécifique au geoweb des flux et des réseaux est examinée d'un point de vue thématique (focalisées sur un jeu de données illustrant un thème), sémiologique ou technologique, au regard des possibilités d'actions sur les différentes composantes de l'image. Ces variables entendent couvrir les différents problèmes généraux liés à la visualisation de l'information scientifique qui ont été présentés par Keim et al. (2008). 


\subsubsection{Un intérêt indéniable pour le support numérique}

L'hypothèse que le développement d'un geoweb spécifique aux flux et réseaux localisés repositionne les cartes dans le paysage géonumérique actuel est confortée par l'augmentation récente du nombre d'applications en ligne que nous avons inventorié. Cette augmentation suggère que le développement de l'informatisation profite effectivement à l'analyse (carto)graphique de flux, réseaux et mouvements de toutes sortes depuis les années 2010 (voir Fig. 2).

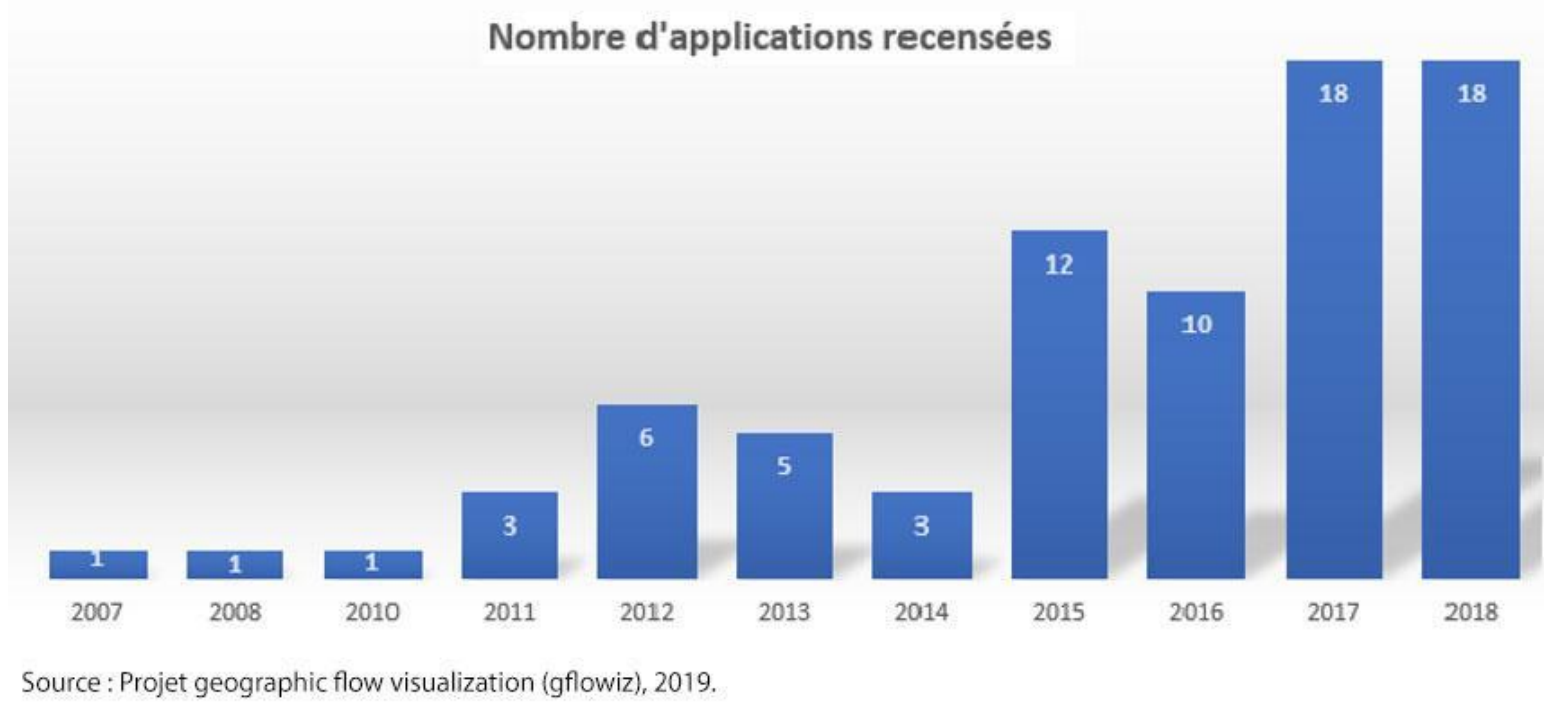

FIGURE 2. Date de mise en ligne des applications recensées

La forte croissance apparente du nombre d'applications recensées est toutefois à nuancer, dans la mesure où il apparaît difficile de réaliser un bilan exhaustif de ces applications, et d'estimer leur part par rapport à un ensemble de sites de cartographie 2.0. La figure 2 décrit donc surtout l'évolution des mises en lignes d'applications web repérées par les contributeurs et contributrices de cette publication, proposant des géovisualisations de données de flux et 
réseaux, sous différentes formes. Le repérage de ces applications se basant en partie sur une veille débutée en 2009 , le « biais du survivant » est ici en partie évité.

Les dispositifs géo numériques utilisés par ces applications sont assez variés. Les applications thématiques, dédiées à un jeu de données particulier, sont les plus nombreuses, avec 50 éléments recensés (sur 80). Ces applications, qui tiennent essentiellement lieu de vitrine, sont cependant souvent fermées. Elles s'opposent aux dispositifs moins fréquents qui permettent d'ajouter des jeux de données externes pour réaliser ses propres cartes : 8 dispositifs de type «logiciels web », ou les mettre en scène : 7 dispositifs de type «storymaps ». Cette diversité témoigne de la levée ces dernières années d'une bonne partie des verrous techniques qui avaient pu être observés empiriquement lors de la première phase de recension, et d'un intérêt grandissant auprès du public pour le support cartographique.

\subsubsection{Des applications monothématiques}

L'un des aspects de la hausse du nombre d'applications répertoriées tient à un effet du contexte de la «crise migratoire » des politiques européennes qui suscita, au cours de ces dernières années, la mise en ligne de nombreuses applications de cartographie de phénomènes migratoires, en réponse aux attentes de la société civile sur ce sujet éminent politique. Il faut ici reconnaître un biais possible d'échantillonnage lié à la spécificité des recherches de la porteuse du projet, Françoise Bahoken, en lien avec le thème des migrations, dans la mesure où c'est elle qui a en très grande partie procédé à la veille et à l'inventaire des applications dont il est question ici. Ces applications sont focalisées pour l'essentiel sur la représentation de ces mouvements de populations réfugiées (prétendument) principalement dirigés vers l'Union européenne (Bahoken et al. 2020). L'augmentation visible à partir de 2015 (du nombre 
d'applications focalisées sur les migrations) sur les années suivantes, serait liée au décalage entre la date de production de l'information, sa mise à disposition et son exploitation cartographique.

Les applications thématiques mobilisant des flux de type démographique, concernant des populations humaines en général, sont donc les plus représentées de notre corpus (avec 20 sur 80). Elles sont ensuite suivies par celles qui décrivent des flux économiques ou scientifiques (10), puis des flux de transport (10). Les 10 applications thématiques restantes sont multithématiques.

Ces variations thématiques influent sur le type de représentation principale (carte de flux ou réseaux origine-destination) et d'implantation spatiale (ponctuelle ou linéaire). Les applications décrivant des liens pondérés, en implantation ponctuelle (bi localisée) sont ainsi plus fréquentes que celles qui mobilisent une implantation (poly)linéaire. Dit autrement, les cartes décrivant des flux origine-destination ordinaires sont plus fréquentes que celles qui sont affectées sur un réseau de transport.

Cela étant, les dispositifs cartographiques dédiés aux mobilités et aux infrastructures de transports sont riches sur le plan de la représentation cartographique (diversité et complexité des modèles graphiques). Les modes concernés sont majoritairement terrestres (ferroviaires et routiers, pour moitié d'entre eux), et, dans une moindre mesure, maritimes ou aériens. Les applications de transport décrivent pour moitié des flots de véhicules en précisant le mode, collectif (bus, métro, navire) ou individuel (voiture, vélos en libre-service). Un petit quart de ces applications de transport concerne enfin des flux de personnes (passagers, migrants, sportifs, etc.). 


\subsection{Une tendance récente : une plus grande flexibilité des applications, vers des outils logiciels web génériques?}

En se concentrant sur les applications les plus récentes du corpus (post-2012), on peut dégager quelques tendances récentes. En particulier, on observe l'ouverture de ces applications web vers plus de généricité, vers une plus grande flexibilité et on assiste même, sur les années les plus récentes, à l'avènement d'outils génériques d'analyse cartographique dans le web. L'une des hypothèses principales qui sous-tend cette tendance réside donc dans la disponibilité de bibliothèques de fonctions qui vont ouvrir un panel de possibilités de graphisme et de cartographie en ligne dès 2011. Le contexte est, en effet, celui d'une convergence entre l'évolution des moteurs javascript des navigateurs et la co-construction d'un "référentiel" commun depuis 2011 qui correspond notamment à la sortie de la version 2 de D3 ${ }^{7}$ et à sa rapide popularisation en 2012 (Bostock et al., 2011), ou encore au lancement de MapBox $\mathrm{G}^{8} \mathrm{~L}$ à partir de 2013.

Ces développements ont fait émerger des opportunités nouvelles pour l'exploration de grands jeux de données. Si l'effort a pendant longtemps été limité à la seule visualisation directe des informations, on observe donc la mise en ligne récente d'applications spécifiquement dédiées à une analyse thématique et interactive configurable par l'utilisateur, qui ouvre la voie vers des approches de statistique descriptive.

Dans notre corpus, on distingue donc d'un côté les applications qui permettent d'explorer de grands jeux de données, mais qui ne permettent pas l'importation de données externes,

\footnotetext{
7 https://d3js.org/

8 https://docs.mapbox.com/mapbox-gl-js/api/
} 
personnelles. C'est le cas de Resource Trade (https://resourcetrade.earth/) par exemple qui s'appuie sur les données ouvertes de la base UN Comtrade (United Nations International trade statistics database) base de données des échanges internationaux déclarés en douane.

L'application Coscimo (http://www.coscimo.net/, 2013) qui permet d'explorer - selon différentes modalités de représentation, à deux périodes et à plusieurs échelles - le réseau mondial des collaborations entre villes, entre aussi dans cette catégorie des applications thématiques permettant d'explorer de grands jeux de données.

D'un autre côté, notre corpus comprend des applications qui autorisent le chargement de données externes, c'est le cas de Flowmap.blue (https://flowmap.blue/, 2018). À cela s'ajoutent parfois des possibilités de co-visualisation comme avec l'outil de visualisation web The Vistorian (https://networkcube.github.io/vistorian/ (2015) adapté à l'exploration de données relationnelles historiques ou encore avec l'application de visualisation et de traitement spatial de données issues de l'activité scientifique, notamment extraites de corpus bibliographiques : Netscity (https://www.irit.fr/netscity/, 2019). Enfin, certaines de ces applications présentent des fonctionnalités avancées de rendu cartographique de flux. On peut par exemple citer le module dédié aux flux de mobilités de France découverte Geoclip Air (https://francedecouverte.geoclip.fr/, 2019).

Pour finir, deux applications sont consacrées à des objectifs de cartographie en ligne. Ces dernières sont révélatrices d'un besoin de renouvellement des pratiques de cartographie tant chez les cartographes thématiciens traditionnels, dans le cas de Magrit (http://magrit.cnrs.fr, 2017), que chez les néo cartographes spécialisés dans le développement web, avec l'exemple de Kepler (https://kepler.gl/, 2018). Il est intéressant de noter que les solutions proposées dans 
les deux cas sont réalisées en lien avec une pratique montante du développement de fonctions voire d'outils libres, reproductibles (Giraut et Lambert, 2017), spécifiquement dédiés à la manipulation de données statistiques à des fins de cartographie thématique.

\section{Typologie des applications web dédiées aux flux et réseaux}

Vu l'importance du corpus d'applications, il nous a semblé intéressant de réaliser une typologie permettant de dégager les principales familles. Plusieurs méthodes statistiques ayant pour objectif de classifier une population en différents sous-groupes peuvent être mobilisées pour cela.

Une classification ascendante hiérarchique $(\mathrm{CAH})$ précédée par une analyse des correspondances multiples $(\mathrm{ACM})$ a été réalisée dans cet objectif. La méthode étant très sensible à la fois aux données manquantes et aux modalités « rares », la liste des variables a été scindée en deux sous-ensembles, toutes n'étant pas encore renseignées de manière exhaustive au moment de l'analyse.

Ainsi, le premier sous-ensemble de variables relève des aspects descriptifs des applications et le second de leurs aspects technologiques - ces derniers feront l'objet d'une typologie spécifique, non présentée ici. La liste détaillant les variables traitées dans la présente contribution est disponible dans l'Annexe 1.

Une première ACM a donc été réalisée sur ce sous-ensemble de variables descriptives. Pour rappel, il s'agit d'une technique d'analyse factorielle qui a pour objectif de résumer l'information contenue dans un grand nombre de variables afin de faciliter l'interprétation de leurs éventuelles corrélations. Sa mise en œuvre conduit finalement à l'identification des 
modalités des applications qui sont corrélées entre elles. Cette première ACM a révélé deux applications très particulières, au sens où elles contribuent fortement $(35,2 \%)$ à la construction du plan factoriel (voir Fig. 3).

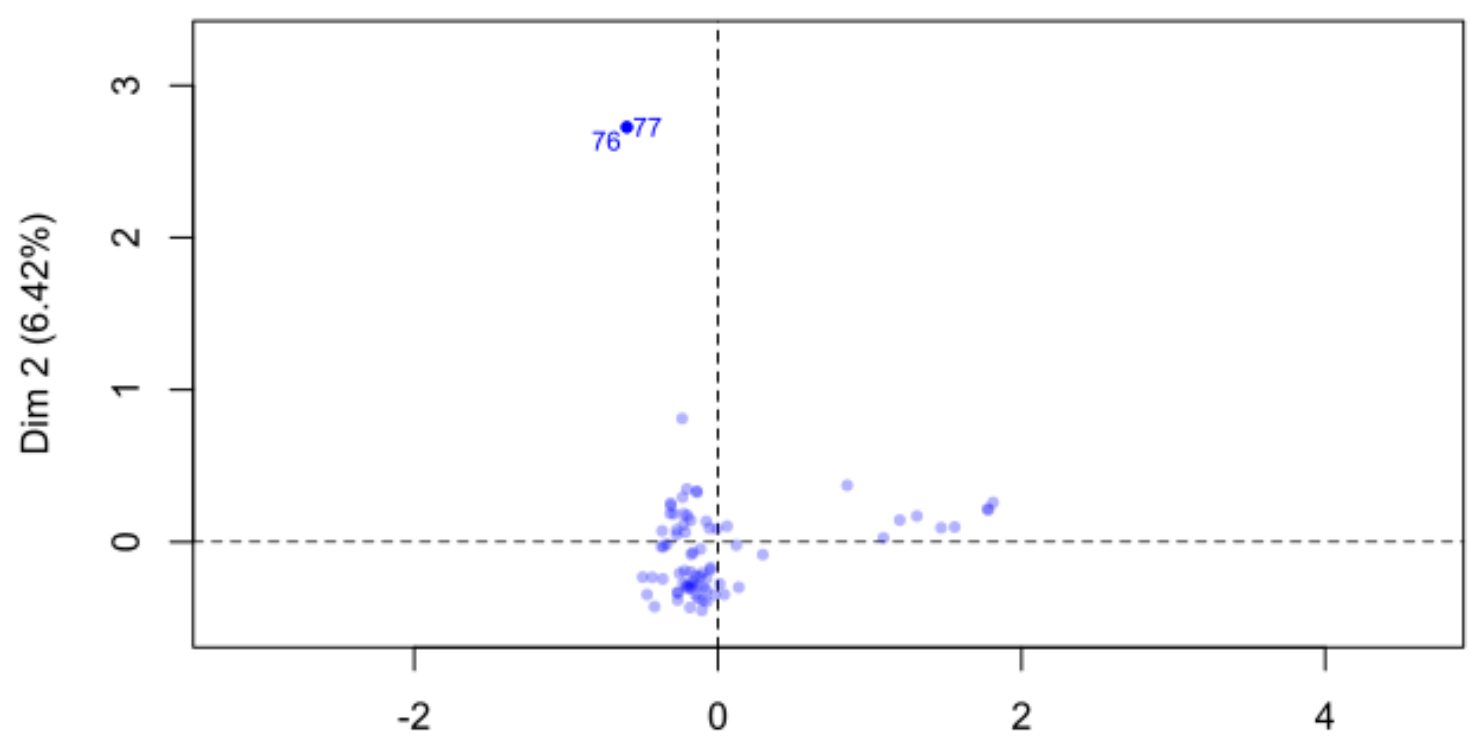

$\operatorname{Dim} 1(7.48 \%)$

FIGURE 3. Contribution des applications de Anagraph à la construction du plan factoriel

Ces deux applications font partie d'un système d'applications développé par la société Anagraph pour décrire 24h du trafic du Grand Montréal à travers deux dispositifs distincts : le premier porte sur des flux: MTL Trajets, identifiant 76 sur la figure 3, https://mtl-

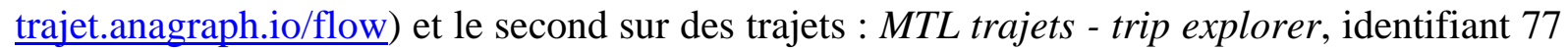
sur la figure 3, https://mtl-trajet.anagraph.io/trips).

Ces deux dispositifs MTL Trajets font figure d'exceptions en raison de leurs très riches caractéristiques. Les modalités qui participent de cette richesse sont les suivantes, présentées par ordre d'importance. La présence 1) d'un fond géo numérique de type OpenStreetMap sur lequel est projeté 2) un déplacement de type flux matériel relevant de 3) tous modes de 
transport. 4) La logique de représentation sous-jacente est celle d'un mouvement qui mobilise dans sa sémiologie 5) des variables rétiniennes dynamiques sous la forme d'animation pour illustrer 6) un transport de personnes. Le dispositif est de type 7) application web (non thématique) déployé à 8) une échelle locale (en intra urbain) sur 9) une seule couche cartographique, 10) en 2018.

Pour analyser au mieux l'ensemble de notre corpus, nous avons choisi d'écarter ces deux cas particuliers avant de relancer l'analyse, ce qui nous conduits à une seconde ACM.

\subsection{Entre réseaux d'acteurs localisés et réseaux planaires}

La seconde ACM apporte des résultats intéressants qui discriminent deux grands types d'applications fondamentalement différentes sur le plan théorique : la première relève de l'analyse de réseaux d'acteurs au sens de la Social network analysis (SNA) et la seconde est typique de l'analyse de réseaux planaires et non planaires. En témoigne la répartition tranchée de nos individus statistiques selon les deux axes de l'ACM : la figure 4 nous montre les individus contribuant le plus à l'élaboration des axes et la figure 5 représente l'ensemble des individus du sous-corpus. 


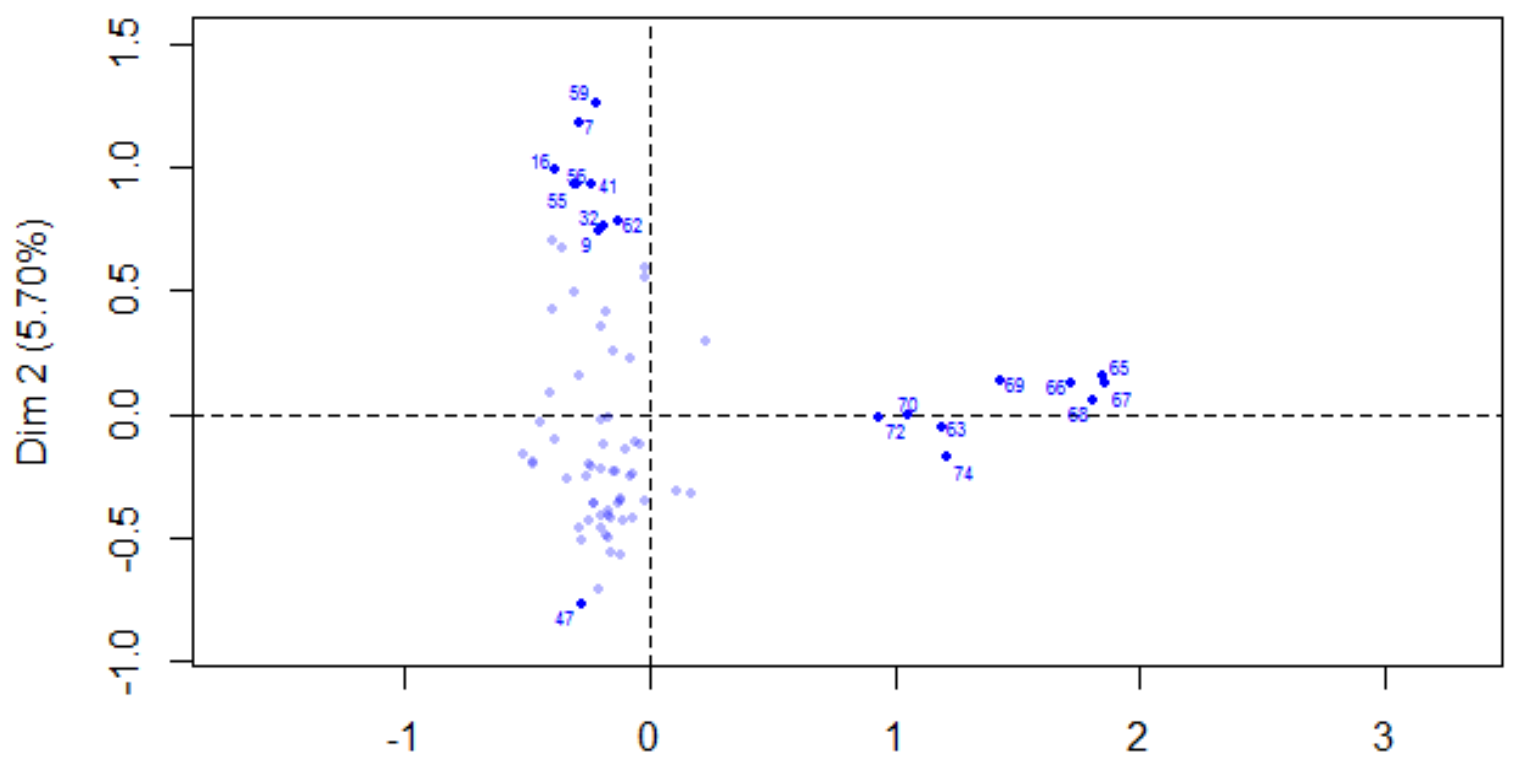

Dim $1(8.18 \%)$

FIGURE 4. Applications ayant la plus forte contribution aux axes

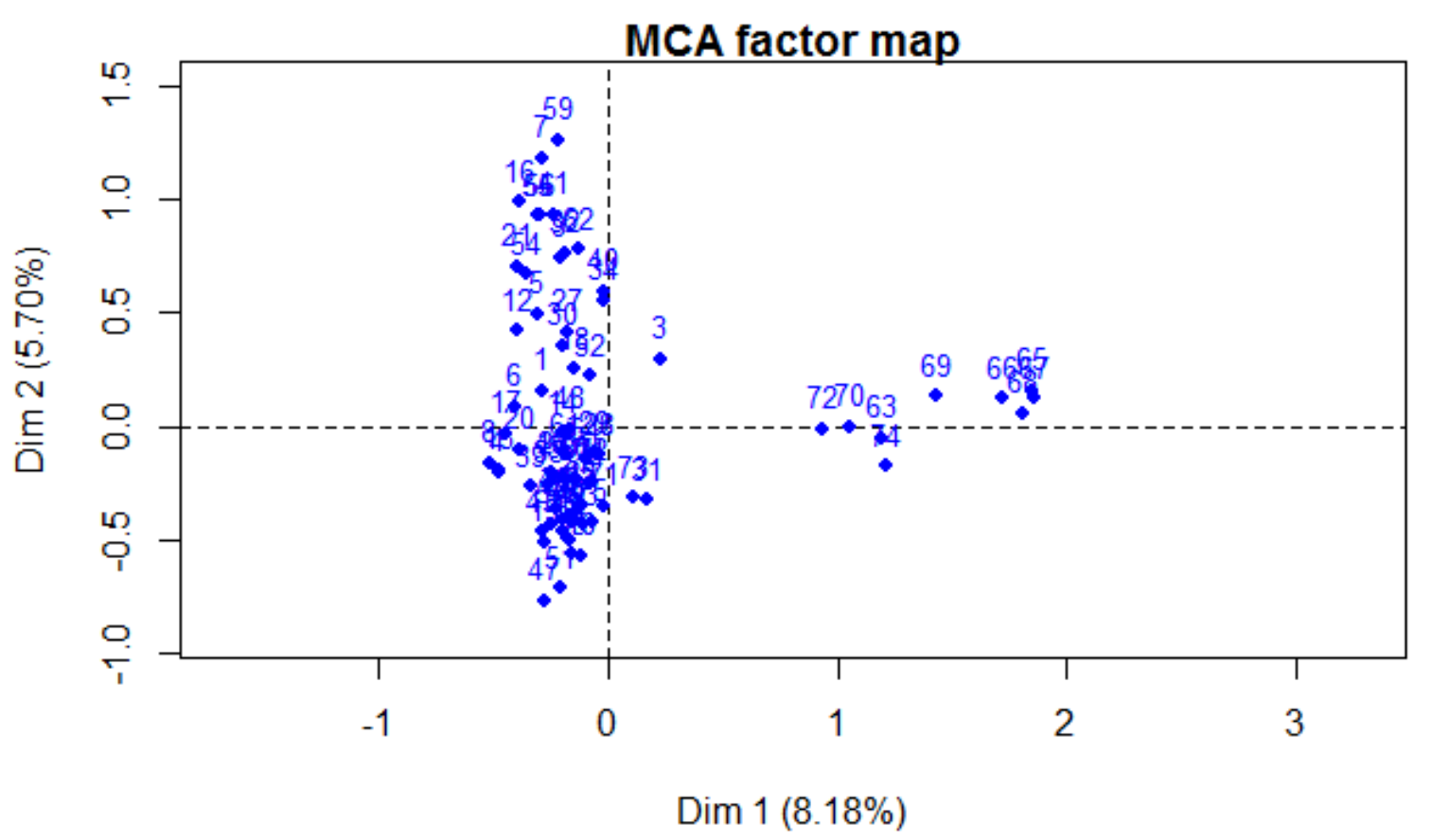

FIGURE 5. Ensemble des individus du sous-corpus 
Une analyse préliminaire de ce sous-corpus d'applications et de variables est réalisée à travers l'examen des variables caractérisant les deux principales dimensions (voir Table 1).

La première dimension de l'ACM apparaît d'abord par le nombre de représentations graphiques distinctes proposées par l'application, puis par le nombre de représentations secondaires et de couches cartographiques, enfin par le type de déplacement représenté. Cette première dimension isole des applications dont la thématique relève plutôt du réseau d'acteurs, en particulier dans le domaine de la représentation des données relationnelles issues de l'activité scientifique avec l'usage de représentations graphiques mixtes pour décrire un lien de type relation scientifique entre lieux. Ici encore, il faut noter un second biais possible d'échantillonnage, lié à la spécialisation de deux membres de notre consortium dans le traitement et la visualisation de données sur la science : Laurent Jégou et Marion Maisonobe.

La deuxième dimension discrimine nos individus sur des applications dont le mode de représentation principale est soit la carte de réseau, soit celle de flux, réalisées respectivement en implantations linéaires / polylinéaires et ponctuelles ; la logique de représentation étant le lien, pondéré ou non, avec des types de déplacements qui opposent matériel (affectés sur un réseau de transport) et immatériel.

\subsection{Trois grandes familles d'applications}

La CAH réalisée sur cette seconde ACM permet d'identifier statistiquement les principales communautés d'applications et de les analyser plus en détail. La meilleure partition obtenue, c'est-à-dire celle qui suggère des groupes où les individus qui les composent sont plus 
homogènes entre eux qu'avec les individus extérieurs, est formée de trois classes qui correspondent aux sous-groupes déjà identifiés lors de l'ACM (voir Fig. 6).

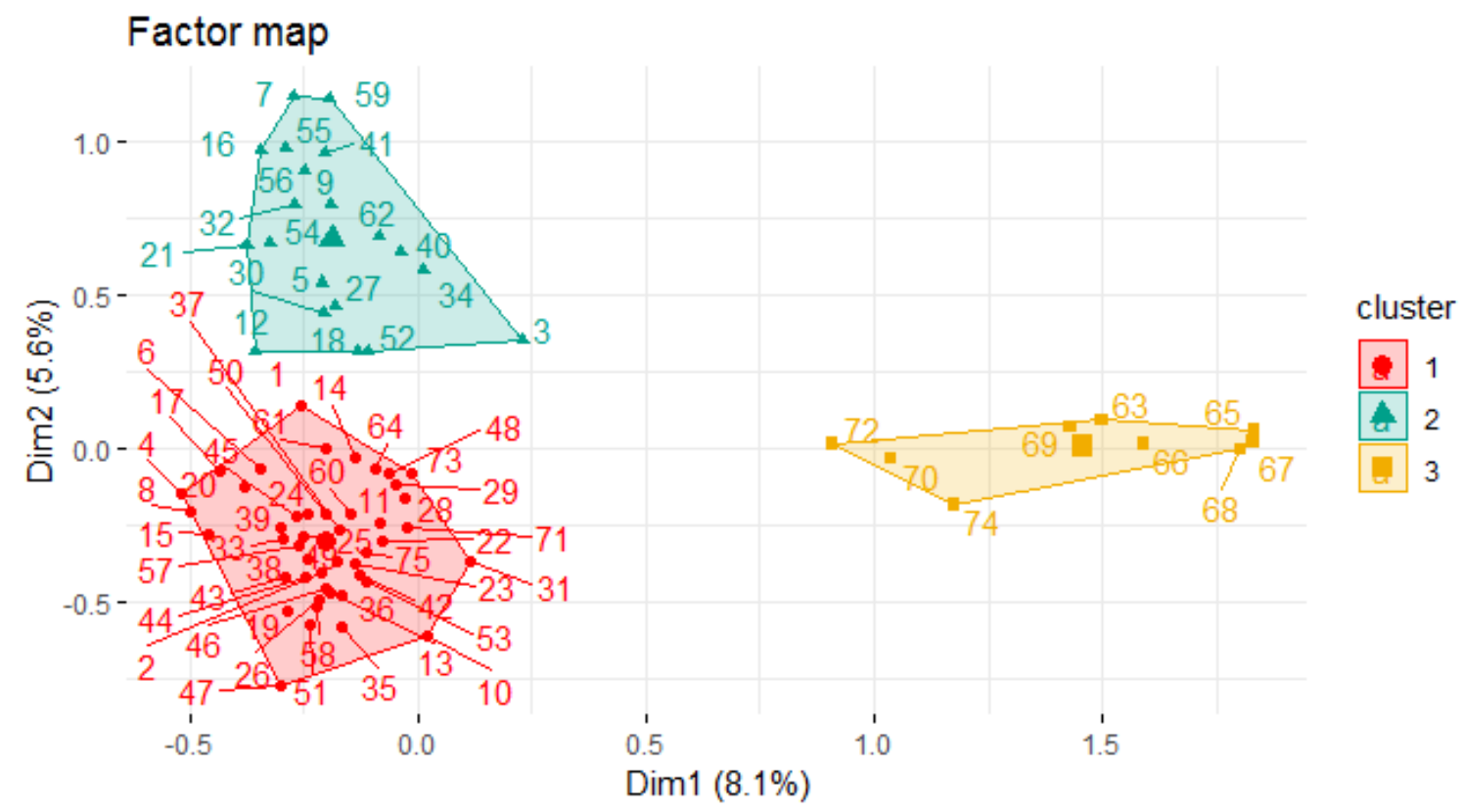

FIGURE 6. Plan des clusters d'applications

Comme l'indiquent Husson et al. (2010), les individus remarquables caractérisant les différents clusters peuvent être classés à l'aide des parangons et des individus « extrêmes ». Les parangons correspondent aux individus «moyens » de chaque classe, situés le plus au centre du nuage de points. Les individus « extrêmes » sont, quant à eux, les plus distants des autres classes.

En plus de l'identification de ces individus particuliers, l'analyse des modalités caractéristiques de chacune des classes apporte une idée précise de la construction de nos clusters, les variables contribuant à la formation de chacun des trois clusters sont présentées dans les sous-sections suivantes. 


\subsubsection{Les applications de type Carte de flux}

Le premier cluster se compose de 46 applications dont la représentation principale est la carte de flux origine-destination (voir Table 2).

Les dispositifs concernés représentent principalement des déplacements immatériels - qui sont figurés sans connaissance du mode de transport -, la carte est souvent accompagnée de plusieurs représentations secondaires et de plusieurs modalités graphiques. Sont également présents des indicateurs statistiques, symbolisés par la variable rétinienne Taille (symboles proportionnels).

Le parangon de cette classe est l'application : Global trade : Africa. The world's import from and export to Africa visualized (https://lab.interactivethings.com/global-trade-africa/), décrivant l'Afrique dans les échanges globaux (exprimés en valeur US\$) de marchandises, en 2012 (voir Fig. 7). Ces flux d'importation et d'exportation mondiaux sont issus de la base UN Comtrade déjà mentionnée : les marchandises y sont regroupées hiérarchiquement selon la Classification type pour le commerce international (CTCI) ${ }^{9}$, établie par les Nations unies pour permettre les comparaisons internationales inter pays sur le commerce extérieur.

\footnotetext{
9 https://ec.europa.eu/eurostat/statistics-explained/index.php/Glossary:Standard_international_trade_classification_(SITC)/fr
} 


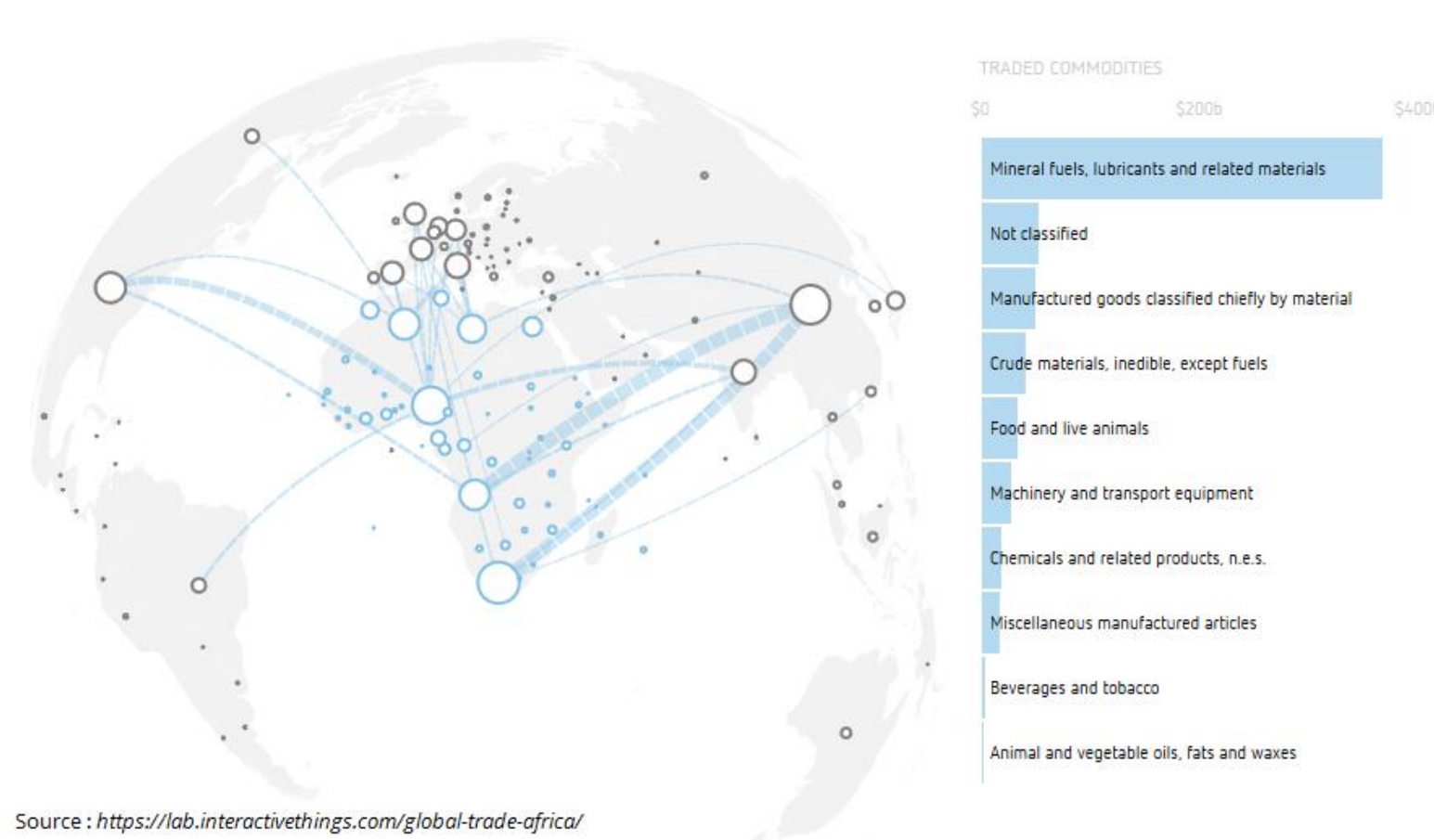

FIGURE 7. Parangon du cluster Carte de flux

L'application Global Trade Africa est particulièrement fluide et interactive. Elle permet d'explorer les données de ces échanges selon plusieurs modalités qui sont géographiques (possibilité de sélection d'un pays ou d'un groupe de pays, en l'occurrence l'Afrique, comme ci-dessus) ou thématiques. Il est en effet possible de naviguer dans la hiérarchie des marchandises de manière de manière à filtrer (ou non) les types de marchandises affichées. Il est par ailleurs possible de visualiser ces marchandises de manière décomposée, en distinguant les importations (en rouge) des exportations (en bleu). La Fig. 7 représente ainsi l'ensemble des groupes de marchandises exportées par des pays africains. 


\subsubsection{Les applications de type réseau planaire}

Le deuxième cluster diffère fortement du précédent, en étant composé plutôt par des applications dont la représentation principale est le réseau, réalisé avec une logique de représentation qui est le lien en implantation polylinéaire (voir Table 3) - alors que dans le cas du cluster précédent, la logique de flux était celle d'une implantation ponctuelle (bi localisée).

On ne trouve pas de calcul d'indicateurs associés (qui conduiraient à pondérer le réseau à l'aide d'une mesure portant sur le trafic ou d'une valeur correspondant au volume de marchandise transporté, par exemple) à ces représentations de type réseau planaire - le type de déplacement étant matériel. Les applications du cluster $n^{\circ} 2$ relèvent surtout du domaine du transport ou de la thématique de l'accessibilité aux réseaux.

Le parangon du cluster Réseau planaire peut être illustré par l'application exploratoire de temps de trajets : Travel Time Tube Map, développée en 2005 par Tom Carden (voir Fig. 8). L'objectif ici est de visualiser la manière dont le plan du métro de Londres se réorganise en fonction de l'heure de départ depuis l'une ou l'autre station. Ainsi au clic sur l'une des stations, les autres se repositionnent suivant une logique de plus court chemin sur le graphe pour finir placées selon des cercles concentriques de 10 minutes, dont le rayon est proportionnel au temps de trajet depuis l'origine. 


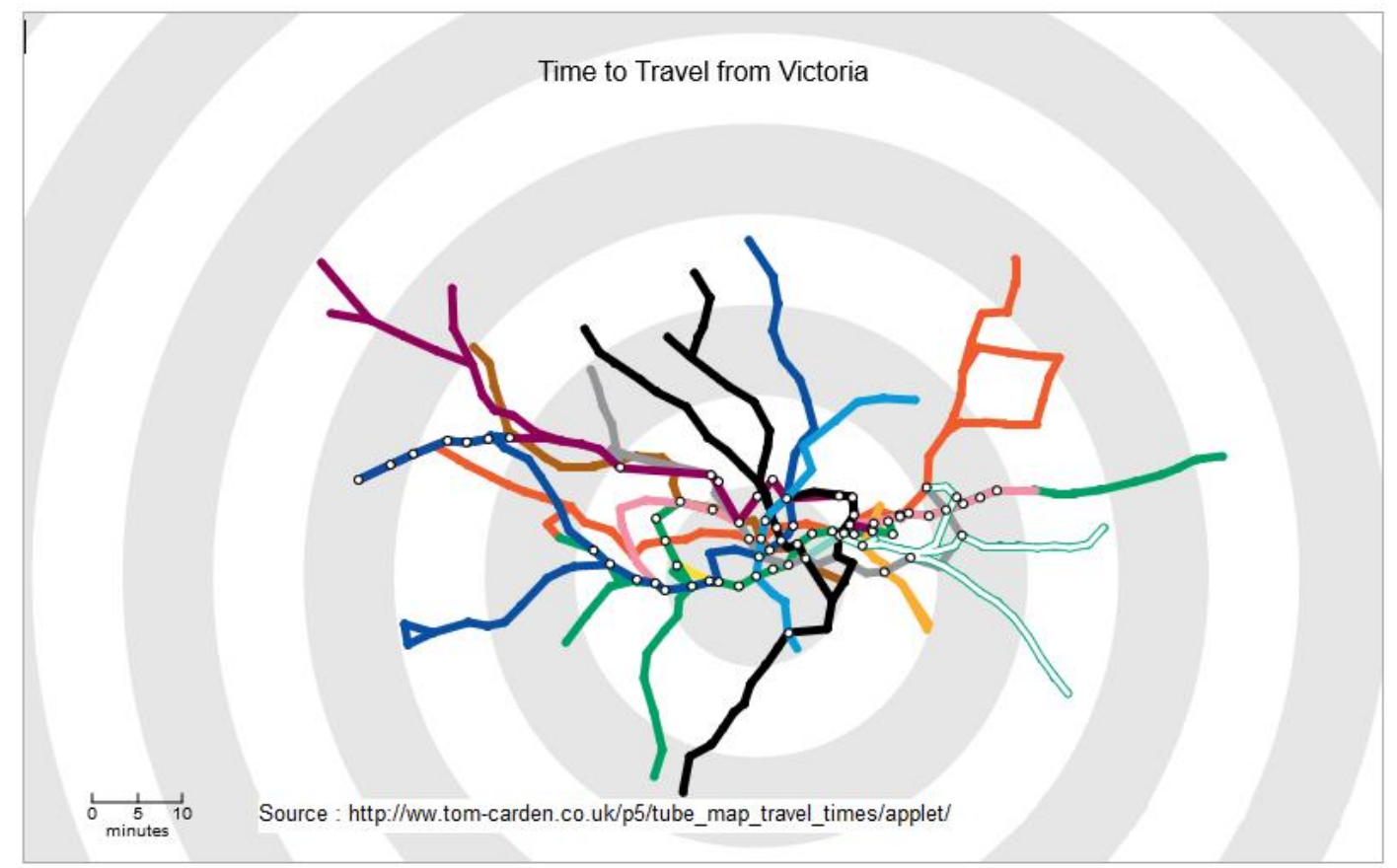

FIGURE 8. Parangon du cluster Réseau planaire

Le résultat est une carte interactive très intuitive qui décrit l'espace-temps d'un parcours en métro et non seulement leur géographie iconique selon le plan-type d'origine, la carte se déformant selon le principe de l'anamorphose vectorielle. Les différentes stations y sont placées en fonction du temps à parcourir pour les rejoindre depuis la station initiale placée au centre de l'image, en l'occurrence Victoria sur la Fig. 8.

\subsubsection{Les applications de type réseau non planaire}

Le troisième cluster est un sous-groupe particulier d'applications, formé de représentations de réseaux non planaires. Les applications qui le composent sont produites exclusivement dans un contexte de recherche (voir Table 4): elles représentent plutôt des réseaux d'acteurs structurés par des relations sociales et notamment scientifique. 
Ce cluster est significativement associé à des représentations mixtes de type graphe, carte et diagramme : les variables rétiniennes Couleur et Taille étant parfois mobilisées, comme ciaprès. Pour ce dernier cluster, le parangon est en effet l'application Palladio : Mapping the Republic of Sciences (http://hdlab.stanford.edu/palladio/) dédié à la visualisation de ses propres données historiques.

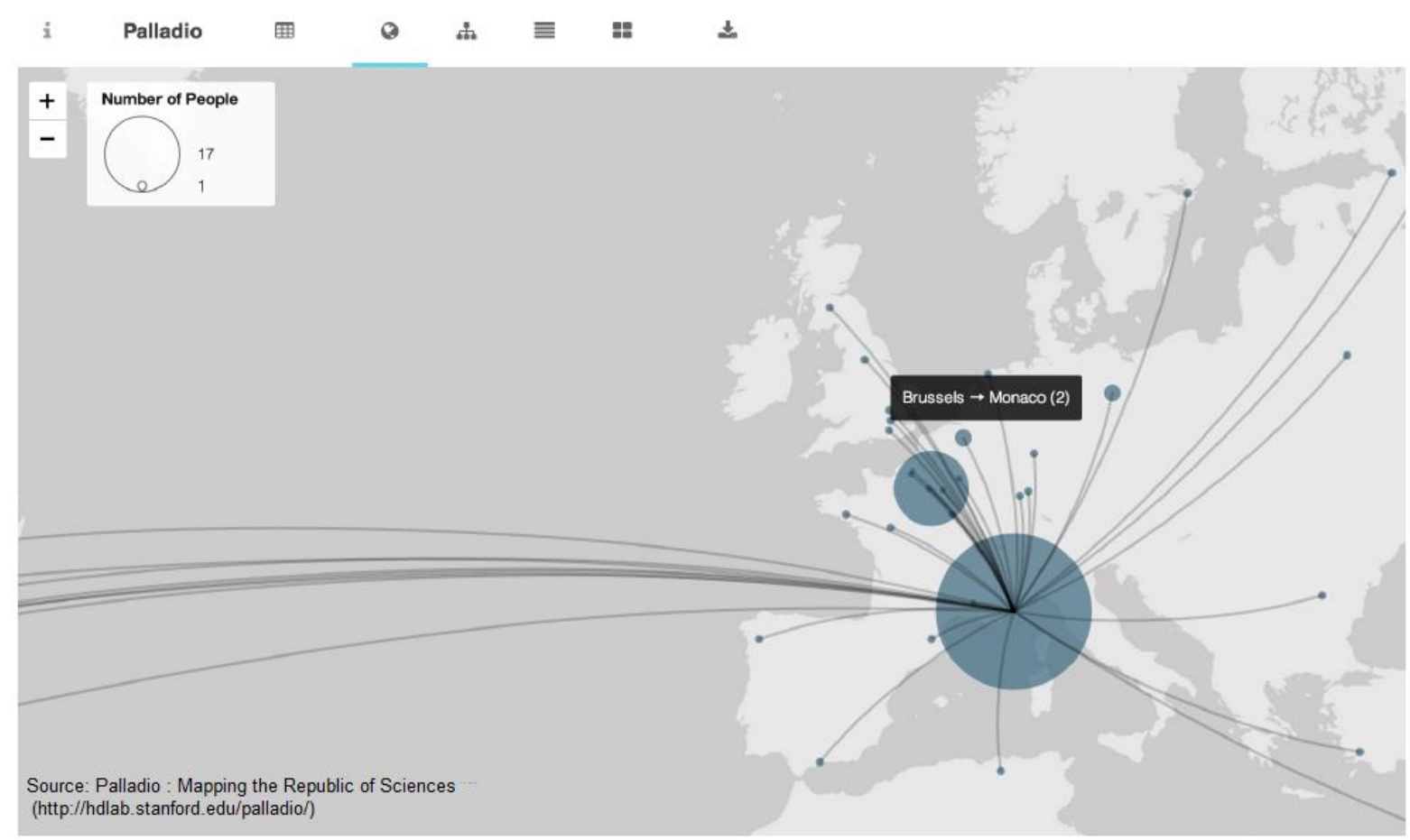

FIGURE 9. Parangon du cluster Réseau non-planaire

Le rendu prend la forme d'une carte décrivant les relations observées du point de vue des liens et aussi de localisations pouvant être pondérées, comme sur la Fig. 9. 


\subsubsection{Les « individus extrêmes»}

Les individus extrêmes de chacun des clusters, ou individus spécifiques, représentent une sorte « d'idéal-type » au sens où ils accentuent des caractéristiques distinctives des applications (voir Table 5).

Ainsi, les individus ou applications spécifiques du cluster 1 sont surtout relatifs aux flux de personnes, essentiellement des migrants sur longues distances ; les applications spécifiques au cluster 2 correspondent aux applications dédiées à l'expression cartographique de mobilités individuelles ou de trafic observés sur des réseaux de transport essentiellement terrestres. Enfin, les applications spécifiques du cluster 3 sont celles qui décrivent des réseaux de relations sociales et notamment scientifiques à l'aide de plusieurs types de représentation (graphe, carte, diagrammes).

\section{Desiderata pour une nouvelle application web}

Les souhaits pour une nouvelle application ont été recueillis dans le cadre d'une Enquête sur les besoins pour une application web de géo-visualisation de flux et de réseaux, menée en ligne au second semestre de 2018. Elle a permis de collecter un peu plus de 200 réponses auprès d'utilisateurs potentiels de l'application, pour apprécier les besoins et les fonctionnalités utiles. Elle nous a servi de support pour la conception d'une nouvelle application, en particulier pour prendre en compte la forte demande en matière d'accès à des outils d'analyse spatiale.

Les répondants sont en grande majorité des personnes qui utilisent déjà des outils d'analyse et de représentation, dans le domaine des transports, de l'enseignement ou de l'environnement, mais qui expriment le besoin de disposer d'un outil plus complet et plus adapté, notamment 
pour créer des représentations interactives et multiples de leurs propres données. Le besoin pour une cartographie de flux, couplée à d'autres représentations (cartes thématiques, graphes et diagrammes) est exprimé dans la majorité des demandes. Les répondants plaident également pour une application qui serait accessible parce qu'en ligne, gratuite, ergonomique et opensource.

La demande de représentations est également importante pour des applications qui soient esthétiquement agréables, basées sur des fonds personnalisables, partageables et diffusables, Ces besoins ne semblent pas comblés par les applications actuelles, de bureau ou en ligne, ce qui justifie la mise en œuvre dans le cadre de notre projet d'une nouvelle offre d'application web pour la cartographie de flux: l'application Arabesque. Cette nouvelle application, présentée pour la première fois à l'occasion de la Conférence internationale de géomatique SAGÉO à Clermont Ferrand en novembre 2019, est libre et gratuite (Côme et al., 2019). L'utilisateur peut y charger ses propres données relationnelles qui sont d'abord mises en forme par défaut, tel qu'illustré par la figure 10 réalisée à partir de données de flux commerciaux internationaux. 


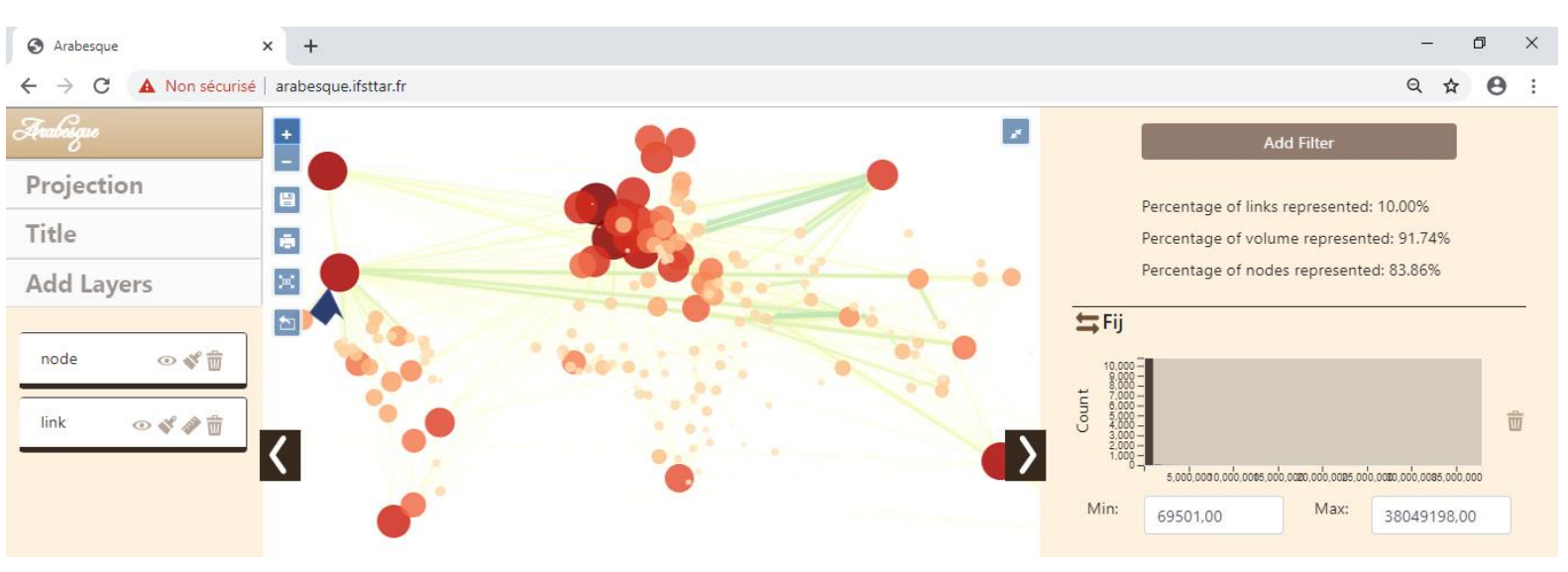

FIGURE 10. Exemple de vue par défaut de l'application Arabesque

L'utilisateur dispose ensuite d'un vaste choix en matière de représentations cartographiques de ses données : choix et stylisation de l'information géographique ; différents fonds de carte peuvent être ajoutés et leur projection cartographique aisément modifiée. Choix et paramétrage des signes et figurés (couleurs, forme, taille des symboles, etc.) dessinés. Filtrage de l'information statistique de flux selon leur intensité ou leur distance parcourue.

Quelques défis restent encore à relever afin d'en faire une application de référence pour la cartographie de flux et de réseaux sur le web : ils tiennent au format d'export de la carte ainsi réalisée en ligne et à l'ajout d'une légende dont l'apparence serait également paramétrable.

\section{Conclusion}

La compilation de près de 80 applications web apporte un éclairage sur les pratiques cartographiques actuelles, en termes de possibilités et de développement.

Si le corpus ainsi constitué apparaît plutôt hétérogène, un premier découpage thématique et méthodologique a permis de dégager trois clusters d'applications. Ces différents groupes d'applications traduisent avant tout des objectifs de représentations fondamentalement 
différents, en lien avec le type d'information mobilisé. Ainsi, les réseaux de relations sociales s'expriment majoritairement sous la forme de graphe (non planaire, immatériel); les déplacements locaux sont représentés sur des cartes de flux affectés ou sous la forme de réseaux planaires, tandis que les déplacements qui s'expriment à plus longue distance sont représentés, quant à eux, sous la forme de cartes de flux origine-destination.

Peu d'applications semblent complètement inscrites dans le paradigme de la « cartographie de visualisation » tel que défini par Mac Eachren (2004). Celles qui ont été recensées ici sont rarement associées à des possibilités de traitement amont des informations statistiques et géographiques. Et pourtant, l'heure semble être au développement de représentations techniquement plus abouties qui laissent entrevoir des possibilités intéressantes sur le support web 2.0. C'est pourquoi le projet gflowiz se propose de travailler à proposer une nouvelle offre à travers le développement et la mise en ligne d'une nouvelle application de cartographie des flux et des réseaux : 1'application Arabesque.

Il est également à noter que les applications de co-visualisation des données selon plusieurs modalités graphiques à l'instar de Coscimo (Jégou et Maisonobe, 2013) ou de The Vistorian (Bach et al., 2015) sont plus rarement proposées, alors que les modes de représentations sont dans bien des cas complémentaires pour explorer des données relationnelles. En particulier, le graphe de flux donne à voir la morphologie d'un système de relations entre des acteurs pouvant être localisés dans un espace, là où la carte décrit leur géographie et le diagramme, leur temporalité.

Le calcul d'indicateurs statistiques complémentaires, par exemple, devant enrichir qualitativement les représentations demeure également largement absent des applications 
inventoriées. Or, ces fonctionnalités sont souvent demandées par les personnes enquêtées, dans le cadre d'outils qui permettraient l'analyse de jeux de données personnels, leur représentation dans des conditions paramétrables, avec une reproductibilité garantie.

Le défi à résoudre dans le cadre du développement d'une nouvelle application web dédiée aux flux et réseaux consiste donc à considérer ces lacunes pour proposer, idéalement, un ensemble d'outils d'analyse et de représentation de matrices d'interactions géographiques qui sont par essence complexes, parce qu'elles sont denses (concernent de nombreux acteurs) et riches, en étant catégorielles et temporelles.

Remerciements : Les résultats présentés ici ont été obtenus dans le cadre de gflowiz, un projet issu d'une collaboration interdisciplinaire entre le CNRS (UMR LISST, UMR Passages et UMR Géographie-cités) et l'Université Gustave Eiffel (départements AME et COSYS), financée par 1'Université Gustave Eiffel et le projet Fédérateur Mobilités et transitions numériques.

\section{Bibliographie}

Bach B., Henry Riche N., Fernandez R., Giannisakis E., Lee B. et Fekete J.-D. 2016. NetworkCube : Bringing Dynamic Network Visualizations to Domain Scientists. In posters of the Conference on Information Visualization (InfoVis). Oct 2015. Chicago.

Bahoken F. 2016. Contribution à la cartographie d'une matrice de flux. Mémoire de Thèse en Géographie - Sciences des territoires, Université Paris Diderot (Paris 7). Paris. Available from https://www.theses.fr/2016USPCC002 [accessed 12 March 2020] 
Bahoken F., Jégou L., Lagarde D. et Lambert N. 2020. La séduction des cartes du geoweb. Le cas des flux de migrants internationaux. Cybergeo : European Journal of Geography. Available from_http://journals.openedition.org/cybergeo/33792 [accessed 12 March 2020]. doi:10.4000/cybergeo.33792.

Baron M., Eckert D. et Jégou, L. 2012. Peut-on démêler l'écheveau mondial des collaborations scientifiques? Revue Mappemonde, Rubrique Internet, $\mathrm{n}^{\circ} 102$. Available from https://mappemonde-archive.mgm.fr/num30/internet/int11201.html [accessed 12 March 2020].

Beauguitte L. 2019. Sur la détection de communautés en général et avec R en particulier, Carnet de recherche du GDR AR-SHS, billet $\mathrm{n}^{\circ} 1314$. Available from https://arshs.hypotheses.org/1314 [accessed 12 March 2020].

Behrisch, M., Bach, B., Henry Riche, N., Schreck, T. et Fekete, J.-D. (2016), Matrix Reordering Methods for Table and Network Visualization. Computer Graphics Forum, 35: pp. 693-716. doi:10.1111/cgf.12935.

Bostock, M., Ogievetsky, V. et Heer, J. 2011. D³ data-driven documents. IEEE transactions on visualization and computer graphics, 17(12), pp.2301-2309.

Côme E., Bapaume T., Jégou L., Bahoken F., Maisonobe M., Roelandt N. et Le Campion G. 2019. Arabesque, application d'exploration et de géovisualisation de données de flux et de réseaux. Actes de la conférence internationale de géomatique (SAGEO’2019), Session stockages et flux, Clermont-Ferrand, 13-15 novembre 2019, pp. 265-264. Available from https://hal.archives-ouvertes.fr/hal-02299349 [accessed 20 may 2020]. 
Crampton J. 2009. Cartography: Maps 2.0. Progress in Human Geography, vol. 33, No.1, pp. 91-100.

Cunty C., Mathian H. et Groupe Cartomouv 2017. Les pratiques de cartographie animée pour représenter le changement. Revue M@ppemonde, 120. Available from http://mappemonde.mgm.fr/120as1/ [accessed 12 March 2020].

Geographic flow vizualisation - gflowiz (2019), Tableau de bord des applications recensées. Available from https://geoflowiz.hypotheses.org/dashboard-des-applications [accessed 04 Novembre 2020].

Giraud, T. et Lambert, N. 2017. Reproducible cartography, Advances in Cartography and GISsciences. Proceedings of the International Cartographic Conference, ICACI'2017, Springer, pp.173-183.

Goodchild M. 2007. Citizens as sensors: Web 2.0 and the volunteering of geographic information. GeoFocus, 7:1-10.

Haklay, M., Singleton, A. et Parker, C. 2008. Web Mapping 2.0: The Neogeography of the GeoWeb. Geography Compass, 2: 2011-2039.

Husson F., Lê S. et Pagès J. 2010. Exploratory Multivariate Analysis by Example Using R. Chapman \& Hall. London.

Joliveau T. 2010. Le GéoWeb pour les Nuls. Available from https://mondegeonumerique.wordpress.com/2010/06/24/le-geoweb-pour-les-nuls/ [accessed 12 March 2020]. 
Joliveau T. 2011. Le géoweb, un nouveau défi pour les bases de données géographiques.

L'Espace géographique, (40) :154-163. Available from https://www.cairn.info/revue-espacegeographique-2011-2-page-154.htm [accessed 12 March 2020].

Joliveau T., Noucher M. et Roche S. 2013. La cartographie 2.0, vers une approche critique d'un nouveau régime cartographique, L'Information géographique, (77):29-46. Available from https://www.cairn.info/revue-l-information-geographique-2013-4-page-29.htm [accessed 12 March 2020].

Keim D., Andrienko G., Fekete J.-D., Görg C., Kohlhammer J. et Melançon G. 2008. Visual Analytics: Definition, Process, and Challenges, In: Kerren A, \& al, (Eds.): Information Visualization, Springer-Verlag, Berlin Heidelberg, LNCS 4950, pp. 154-175.

Le Campion G. et Nguyen A. 2019. gFlowiz DB : tableau de bord d'exploration interactive d'applications web de visualisation de flux et mouvements, Université Paris-Est, AMESPLOTT / COSYS-GRETTIA, IFSTTAR et UMR Passages.

Mac Eachren A. 2005. How Maps Work, Representation, Visualization, and Design. The Guildford Press, New-York.

Mericskay B. et Roche S. 2011. Cartographie 2.0 : le grand public, producteur de contenus et de savoirs géographiques avec le web 2.0. Cybergeo : European Journal of Geography. Available from http://journals.openedition.org/cybergeo/24710 [accessed 15 may 2020].

Noucher M. 2017. Les petites cartes du web. Approches critiques des nouvelles fabriques cartographiques. Éditions de la rue d'Ulm - Presses de l'École normale supérieure, Paris. 
Sui D., Elwood S. et M. Goodchild (dir.) 2012. Crowdsourcing Geographic Knowledge : Volunteered Geographic Information (VGI) in Theory and Practice, Springer, New York.

Turkucu A. et Roche S. 2008. Classification fonctionnelle des Public Participation GIS. Revue internationale de géomatique. 18 (3-4), pp. 11-24.

Zanin C. 2013. Cartographie et analyse territoriale. Mémoire d'habilitation à diriger des recherches en Géographie, Université Paris Diderot (Paris 7). Paris. 
TABLE 1. Liste des variables - voir descriptif dans l'Annexe 2 caractérisant les deux dimensions de l'ACM

\begin{tabular}{|l|c|c|}
\hline \multicolumn{1}{|c|}{ DIMENSION 1 } & $\mathbf{R}^{\mathbf{2}}$ & p. value \\
\hline Nb. de représentations & 0,905 & $8,67 \mathrm{e}-33$ \\
graphiques & & $3,72 \mathrm{e}-14$ \\
\hline Nb. de représentations & 0,641 & \\
graphiques secondaires & & $7,49 \mathrm{e}-26$ \\
\hline Thématique & 0,766 & $5,23 \mathrm{e}-24$ \\
\hline Logique de représentation & 0,585 & \\
\hline
\end{tabular}

\begin{tabular}{|l|c|c|}
\hline \multicolumn{1}{|c|}{ DIMENSION 2 } & $\mathbf{R}^{\mathbf{2}}$ & p. value \\
\hline Implantation polylinéaire & 0,589 & $9,04 \mathrm{e}-16$ \\
\hline $\begin{array}{l}\text { Type de représentation } \\
\text { principale }\end{array}$ & 0,641 & $3,72 \mathrm{e}-14$ \\
\hline Thématique & 0,766 & $4,90 \mathrm{e}-14$ \\
\hline Logique de représentation & 0,585 & $9,09 \mathrm{e}-13$ \\
\hline
\end{tabular}


TABLE 2. Liste des variables - voir descriptif dans l'Annexe 3 formant le cluster 1 : Carte de flux

\begin{tabular}{|c|c|c|c|c|c|}
\hline Variable & Cla/Mo & $\begin{array}{l}\text { Mod/Cl } \\
\text { a }\end{array}$ & Global & p.value & v.test \\
\hline Implantation_lineaire_OUI & 97,14 & 73,91 & 46,66 & $\begin{array}{l}3,92 \mathrm{e}- \\
10\end{array}$ & 6,25 \\
\hline $\begin{array}{l}\text { Type_representation_principale_Cart } \\
\text { e de flux }\end{array}$ & 85,41 & 89,13 & 64 & $\begin{array}{l}1,25 \mathrm{e}- \\
08\end{array}$ & 5,69 \\
\hline Indicateur_OUI & 87,80 & 78,26 & 54,66 & $\begin{array}{l}2,14 \mathrm{e}- \\
07\end{array}$ & 5,18 \\
\hline Logique_representation_Flux & 83,33 & 76,08 & 56 & $\begin{array}{l}1,22 \mathrm{e}- \\
05\end{array}$ & 4,37 \\
\hline Type_depl_immateriel & 90 & 58,69 & 40 & $\begin{array}{l}2,24 \mathrm{e}- \\
05\end{array}$ & 4,23 \\
\hline VV_taille_OUI & 85,29 & 63,04 & 45,33 & 0,0001 & 3,88 \\
\hline Nb_representations_secondaires_2 & 100 & 34,78 & 21,33 & 0,0001 & 3,85 \\
\hline Domaine_Demographie & 94,73 & 39,13 & 25,33 & 0,0003 & 3,60 \\
\hline Nb_representations_graphiques_3 & 100 & 26,08 & 16 & 0,001 & 3,17 \\
\hline VVD_OUI & 89,47 & 36,95 & 25,33 & 0,003 & 2,96 \\
\hline
\end{tabular}


TABLE 3. Liste des variables - voir descriptif dans l'Annexe 4-

formant le cluster 2 : Réseau planaire

\begin{tabular}{|c|c|c|c|c|c|}
\hline & Cla/Mod & $\mathrm{Mod} / \mathrm{Cla}$ & Global & p,value & $\mathrm{v}$,test \\
\hline Implantation_polylineaire_OUI & 100 & 80 & 21,33 & $\begin{array}{c}5,66 \mathrm{e}- \\
13\end{array}$ & 7,20 \\
\hline $\begin{array}{l}\text { Type_representation_principale_Cart } \\
\text { e de reseau }\end{array}$ & 100 & 80 & 21,33 & $\begin{array}{c}5,66 \mathrm{e}- \\
13\end{array}$ & 7,20 \\
\hline Logique_representation_Liens & 100 & 55 & 14,66 & $\begin{array}{c}3,42 \mathrm{e}- \\
08\end{array}$ & 5,51 \\
\hline Implantation_lineaire_NON & 47,5 & 95 & 53,33 & $\begin{array}{c}6,06 \mathrm{e}- \\
06\end{array}$ & 4,52 \\
\hline Indicateur_NON & 50 & 85 & 45,33 & $\begin{array}{c}3,56 \mathrm{e}- \\
05\end{array}$ & 4,13 \\
\hline Logique_representation_Autre & 100 & 30 & 8 & 0,0001 & 3,72 \\
\hline Domaine_Transport & 54,54 & 60 & 29,33 & 0,0009 & 3,31 \\
\hline Mode_de_transport_Ferroviaire & 85,71 & 30 & 9,33 & 0,001 & 3,25 \\
\hline Type_depl_materiel & 64,28 & 45 & 18,66 & 0,001 & 3,21 \\
\hline Thematique_Accessibilite au reseau & 100 & 20 & 5,33 & 0,003 & 2,87 \\
\hline
\end{tabular}


TABLE 4. Liste des variables - voir descriptif dans l'Annexe 5 formant le cluster 3 : Réseau non planaire

\begin{tabular}{|c|c|c|c|c|c|}
\hline & $\begin{array}{l}\text { Cla/Mo } \\
\text { d }\end{array}$ & Mod/Cla & Global & p,value & $\begin{array}{l}\mathrm{v}, \text { tes } \\
\mathrm{t}\end{array}$ \\
\hline Type_representation_principale_Mixte & 100 & 66,66 & 8 & $\begin{array}{c}4,17 \mathrm{e}- \\
07\end{array}$ & 5,06 \\
\hline Thematique_Reseau d'acteurs & 100 & 55,55 & 6,66 & $\begin{array}{c}7,30 \mathrm{e}- \\
06\end{array}$ & 4,48 \\
\hline Population_Relations scientifiques & 100 & 44,44 & 5,33 & 0,0001 & 3,88 \\
\hline Type_depl_NC & 100 & 44,44 & 5,33 & 0,0001 & 3,88 \\
\hline $\begin{array}{l}\text { Contexte_de_developpement=Recherch } \\
\text { e }\end{array}$ & 50 & 66,66 & 16 & 0,0003 & 3,60 \\
\hline VV_couleur_NON & 32 & 88,88 & 33,33 & 0,0004 & 3,50 \\
\hline Echelle=Echelle_NC & 80 & 44,44 & 6,66 & 0,0004 & 3,48 \\
\hline Domaine=Domaine_Science & 80 & 44,44 & 6,66 & 0,0004 & 3,48 \\
\hline Domaine=Domaine,NA & 100 & 33,33 & 4 & 0,001 & 3,22 \\
\hline implantation_lineaire_NON & 22,5 & 100 & 53,33 & 0,002 & 3,06 \\
\hline VV_taille_NON & 21,95 & 100 & 54,66 & 0,002 & 2,99 \\
\hline
\end{tabular}


TABLE 5. Individus spécifiques des trois clusters

\begin{tabular}{|c|c|c|}
\hline Cluster 1 & Cluster 2 & Cluster 3 \\
\hline Flow map Explorer & Strava & Netscity \\
\hline EU terre d'accueil des nobélisés & Carto.metro.tramwa & Palladio \\
migrants & y & \\
\hline Flux de mobilité tous modes & OpenRailwayMap & Cortext Platform \\
\hline 2013 de Toulouse & Mapple & Coscimo \\
\hline People moving & WWF Arctic & The Vistorian \\
\hline Torflow & Geographical & \\
\hline
\end{tabular}




\section{Figure captions}

FIGURE 1. Tableau de bord des applications recensées

FIGURE 2. Date de mise en ligne des applications recensées

FIGURE 3. Contribution des applications de Anagraph à la construction du plan factoriel

FIGURE 4. Applications ayant la plus forte contribution aux axes

FIGURE 5. Ensemble des individus du sous-corpus

FIGURE 6. Plan des clusters d'applications

FIGURE 7. Parangon du cluster Carte de flux

FIGURE 8. Parangon du cluster Réseau planaire

FIGURE 9. Parangon du cluster Réseau non-planaire

FIGURE 10. Exemple de vue par défaut de l'application Arabesque 


\section{Annexe 1. Liste des variables du sous-corpus d'application analysés}

Les variables décrivant le sous-groupe d'applications qui a fait l'objet de l'ACM et de la CAH présentées dans le texte sont les suivantes : le titre, le nombre de couches cartographiques (de 1 à 231), le nombre de représentations graphiques (de 1 à 7), le dispositif géo-numérique (java, web (collecte), web thématique, web interactif, storymap, prototype, logiciel web, package $\mathrm{R}, \ldots$ ), le type de représentation principale (carte de flux, carte de réseau, graphe, diagramme à coordonnées parallèles), la logique de représentation (lieux, liens, flux, réseaux), l'implantation spatiale (ponctuelle, linéaire, polylinéaire, surfacique), le mode de représentation (vecteur et/ou raster, mixte), l'indicateur statistique représenté, la présence d'une variable rétinienne dynamique ou non, de la variable visuelle Taille et/ou de la teinte (Couleur) incluant l'opacité et l'opposition colorée et/ou de la Forme et/ou du Clignotement et/ou de l'Animation et/ou du Mouvement, le nombre de représentations secondaires, la présence d'une légende, le langage logiciels (Javascript, MapBox, PHP, Python, ...) le nombre de dimensions spatiales (1D, 2D, 3D, 3D globe), le fonds géonumérique (OSM, Mapbox, Thunder Forest, BingMaps, Google Maps, ... ), le public visé, la date de réalisation, le contexte de développement (recherche, public, privé), le domaine (économie, démographie, environnement, littérature, politique, transports, télécommunication, santé, ...), l'échelle géographique, la thématique (mobilités, migrations, sport, armement, ...), le type de déplacement, la population (statistique) concernée et le mode de transport. 
Annexe 2. Descriptif des variables de la Table 1 caractérisant les deux dimensions de l'ACM.

$\mathrm{Nb}$. de représentations graphiques : nombre total d'images.

$\mathrm{Nb}$. de représentations graphiques secondaires : nombre total de second plan.

Thématique : sujet de l'application (ex. transport, migrations).

Logique de représentation : raisonnement théorique sous-jacent (représentation de liaisons OD, de flux (liaisons pondérées) ou de mouvements (liaisons pondérées et prenant en compte une variable spatiale).

Implantation polylinéaire : implantation spatiale linéaire formée d'au moins trois points, décrivant une trajectoire (objet équivalent à un graphe planaire).

Type de représentation principale : modalité du type d'image principale (graphe, matrice ordonnée, carte de flux, coordonnées parallèles, ...). 


\section{Annexe 3. Descriptif des variables et des résultats présentés sur la Table 2 caractérisant la formation du cluster 1 : Carte de flux}

\section{Description des variables}

Implantation linéaire : implantation spatiale linéaire formée de deux points au maximum, décrivant un lien ordinaire ou un lien pondéré (flux) entre une origine et une destination. Type_representation_principale_Carte de flux : l'image principale est une carte de flux OD. Indicateur_OUI : les flux représentés ont été préalablement transformés en indicateurs (ex. balance des échanges)

Logique_representation_Flux : le raisonnement théorique sous-jacent conduit à la représentation de liaisons origine-destination pondérées.

Type_depl_immateriel : le type de déplacement ne considère pas un mode de transport. VV_taille_OUI : l'application mobilise dans la sémiologie de l'image principale la variable rétinienne taille

Nb_representations_secondaires_2 : outre l'image principale, deux autres images sont présentées

Domaine_Demographie : l'application relève du champ disciplinaire général démographie. Nb_representations_graphiques_3 : l'application propose au total trois images VVD_OUI : 1'application mobilise dans sa sémiologie des variables visuelles dynamiques, qui sont spécifiques au support écran (exemple : clignotement, transparence).

Description des résultats

Cla/Mod : pourcentage d'individus possédant la modalité qui appartiennent à la classe Mod/Cla : pourcentage des individus de la classe qui prennent la modalité 
Global : pourcentage global des individus (i.e. de toute la population) qui prennent la modalité p-value : significativité de la différence entre les proportions Mod/Cla et Global v.test: une mesure d'association entre variables, mesure l'association de la modalité avec la classe 


\section{Annexe 4. Descriptif des variables et des résultats présentés sur la Table 3 caractérisant la formation du cluster 2 : Réseau planaire}

\section{Description des variables}

Implantation polylinéaire : implantation spatiale linéaire formée d'au moins trois points, décrivant une trajectoire (objet équivalent à un graphe planaire).

Type_representation_principale_Carte de réseau : l'image principale est une carte de réseau planaire.

Logique_representation_Liens : le raisonnement théorique sous-jacent conduit à la représentation de liaisons non pondérées, sous la forme de liens.

Implantation_lineaire_NON : implantation spatiale non linéaire, elle est potentiellement ponctuelle ou aréale.

Indicateur_NON : les liaisons éventuellement représentées n'ont pas fait l'objet d'une transformation en indicateurs, l'image représente les valeurs observées.

Logique_representation_Autre : le raisonnement théorique sous-jacent ne relève pas de la représentation de liaisons au sens général

Domaine_Transport : l'application relève du champ des transports.

Mode_de_transport_Ferroviaire : l'application mobilise un mode de transport par voie ferrée. Type_depl_materiel : le type de déplacement considère un mode de transport, qui sert de support à la représentation

Thematique_Accessibilite au reseau : l'application relève des problématiques d'accessibilité (à ou grâce à un réseau de transport)

Description des résultats 
Cla/Mod : pourcentage d'individus possédant la modalité qui appartiennent à la classe

Mod/Cla : pourcentage des individus de la classe qui prennent la modalité

Global : pourcentage global des individus (i.e. de toute la population) qui prennent la modalité p-value : significativité de la différence entre les proportions Mod/Cla et Global v.test: une mesure d'association entre variables, mesure l'association de la modalité avec la classe 


\section{Annexe 5. Descriptif des variables et des résultats présentés sur la Table 4 caractérisant la formation du cluster 3 : Réseau non planaire}

\section{Descriptif des variables}

Type_representation_principale_Mixte : la vue principale combine plusieurs types d'images (eemple : exemple : un graphe et une carte de flux origine-destination).

Thematique_Reseau d'acteurs : l'application relève des problématiques spécifiques à l'analyse de réseaux d'acteurs sociaux, au sens de la Social Network analysis.

Population_Relations scientifiques : la population, au sens statistique, qui est représentée est la relation scientifique.

Type_depl_NC : le type de déplacement (qui induit en filigrane un mode de transport) n'est pas une variable pertinente.

Contexte_de_developpement=Recherche : l'application a été développée dans un institut de recherche.

VV_couleur_NON : l'application ne mobilise pas dans sa sémiologie la variable rétinienne couleur.

Echelle=Echelle_NC : 1'application ne propose pas de composante d'échelle géographique. Domaine=Domaine_Science : l'application relève du champ disciplinaire d'étude des sciences (histoire, géographie, etc.).

Domaine=Domaine,NA : l'application ne relève pas d'un champ disciplinaire spécifique. implantation_lineaire_NON : implantation spatiale non linéaire, elle est potentiellement ponctuelle ou aréale.

VV_taille_NON : l'application ne mobilise pas dans sa sémiologie la variable rétinienne taille. 
Descriptif des résultats

Cla/Mod : pourcentage d'individus possédant la modalité qui appartiennent à la classe

Mod/Cla : pourcentage des individus de la classe qui prennent la modalité

Global : pourcentage global des individus (i.e. de toute la population) qui prennent la modalité p-value : significativité de la différence entre les proportions Mod/Cla et Global v.test: une mesure d'association entre variables, mesure l'association de la modalité avec la classe 